\title{
ON THE SIMULTANEOUS APPROXIMATION OF TWO REAL NUMBERS ${ }^{1}$
}

\author{
RAPHAEL M. ROBINSON
}

If $\xi_{1}, \xi_{2}, \cdots, \xi_{n}$ are any real numbers and $t$ is a positive integer, then it is well known that integers $a_{1}, a_{2}, \cdots, a_{n}, b$ can be found, such that $0<b \leqq t^{n}$ and

$$
\left|b \xi_{k}-a_{k}\right|<1 / t, \quad k=1,2, \cdots, n .
$$

The proof is briefly the following. ${ }^{2}$ Consider the $t^{n}+1$ points $\left(r \xi_{1}, r \xi_{2}, \cdots, r \xi_{n}\right)$, where $r=0,1, \cdots, t^{n}$. Reduce mod 1 to congruent points in the unit cube $\left(0 \leqq x_{1}<1, \cdots, 0 \leqq x_{n}<1\right)$. If this cube is divided in to $t^{n}$ cubes of edge $1 / t$ (including the lower boundaries), then at least one of these small cubes must contain two of the reduced points, say those with $r=r^{\prime}$ and $r=r^{\prime \prime}$. With $b=\left|r^{\prime}-r^{\prime \prime}\right|$ and suitable $a$ 's, we evidently satisfy the required inequalities.

For $n=1$, the inequality can be sharpened to

$$
|b \xi-a| \leqq 1 /(t+1),
$$

$b$ satisfying the condition $0<b \leqq t$. For if we consider the points $r \xi$ $(r=0,1, \cdots, t)$, and mark the points in the interval $0 \leqq x \leqq 1$ which are congruent to them mod 1 , we have at least $t+2$ points marked, since corresponding to $r=0$ we mark both 0 and 1 . Some two of the marked points must lie within a distance $1 /(t+1)$ from each other, so that the desired conclusion follows. This is the best result, as the example $\xi=1 /(t+1)$ shows.

The present note solves the corresponding problem for $n=2$. For larger values of $n$ the problem appears more difficult.

THEOREM. If $\xi_{1}$ and $\xi_{2}$ are any real numbers, and $s$ is a positive integer, then integers $a_{1}, a_{2}, b$ can be found, such that $0<b \leqq s$, and

$$
\left|b \xi_{k}-a_{k}\right| \leqq \max \left(\frac{\left[s^{1 / 2}\right]}{s+1}, \frac{1}{\left[s^{1 / 2}\right]+1}\right), \quad k=1,2 .
$$

For every $s$, values of $\xi_{1}$ and $\xi_{2}$ can be found for which the inequalities could not both be satisfied if the equality sign were omitted.

\footnotetext{
1 Presented to the Society, November 23, 1940.

2 The method used in this proof (Schubfachprinzip or "pigeonhole principle") was first used by Dirichlet in connection with a similar problem. We sketch the proof here in order to compare it with the proof of the theorem below, which also uses that method.
} 
The inequalities may also be written

$$
\left|b \xi_{k}-a_{k}\right| \leqq \begin{cases}t /(s+1) & \text { for } t^{2}-1 \leqq s \leqq t(t+1)-1 \\ 1 /(t+1) & \text { for } t(t+1)-1 \leqq s \leqq(t+1)^{2}-1\end{cases}
$$

It will be noted that in some intervals the bound does not decrease as $s$ increases.

We show first that the theorem is the best possible. We shall think of the inequalities in the form just given. If $s<(t+1)^{2}$, then it is evident that $\xi_{1}=1 /(t+1), \xi_{2}=1 /(t+1)^{2}$ are a pair of real numbers which cannot be approximated simultaneously with an error less than $1 /(t+1)$; this settles the second case. For the first case, consider the pair of real numbers $\xi_{1}=1 /(s+1), \xi_{2}=t /(s+1)$. We are to show that not both errors can be made less than $t /(s+1)$. We note first that $b \xi_{1}$ and $b \xi_{2}$ differ from integers by the same amount as $(s+1-b) \xi_{1}$ and $(s+1-b) \xi_{2}$; hence we may suppose that $b \leqq(s+1) / 2$, and therefore $0<b \xi_{1} \leqq 1 / 2$. In order to make $\left|b \xi_{1}-a_{1}\right|<t /(s+1)$, we must have $0<b<t$. Then $0<b \xi_{2}<1$. Since $b \xi_{2} \geqq \xi_{2}=t /(s+1)$ and $1-b \xi_{2} \geqq$ $1-(t-1) \xi_{2}=1-(t-1) t /(s+1) \geqq t /(s+1)$, we see that the inequality $\left|b \xi_{2}-a_{2}\right|<t /(s+1)$ cannot be satisfied.

The theorem evidently follows from the lemma below, by putting $t=\left[s^{1 / 2}\right]$.

LEMmA. Let $s$ and $t$ be positive integers with $s \geqq t$. If $\xi_{1}$ and $\xi_{2}$ are any real numbers, then integers $a_{1}, a_{2}, b$ can be found, such that $0<b \leqq s$, and

$$
\left|b \xi_{1}-a_{1}\right| \leqq t /(s+1), \quad\left|b \xi_{2}-a_{2}\right| \leqq 1 /(t+1) .
$$

PROOF. Consider the points $\left(r \xi_{1}, r \xi_{2}\right)$ with $r=0,1, \cdots, s$. Mark all the points congruent to these mod 1 which fall in the rectangle $0 \leqq x_{1} \leqq t, 0 \leqq x_{2}<1$. There are $(s+1) t$ points to be marked with $x_{1}<t$; and in addition, the point $(t, 0)$ is marked, corresponding to $r=0$. If we divide our rectangle into $s+1$ rectangles of width $t /(s+1)$ (closed except at the top) by means of vertical lines, then at least one of them contains more than $t$ points, all corresponding to different values of $r$. The corresponding values of $x_{2}$ are $t+1$ or more numbers, some two of which differ mod 1 by not more than $1 /(t+1)$. Thus we find two points $\left(r^{\prime} \xi_{1}, r^{\prime} \xi_{2}\right)$ and $\left(r^{\prime \prime} \xi_{1}, r^{\prime \prime} \xi_{2}\right)$, whose horizontal distance mod 1 does not exceed $t /(s+1)$ and whose vertical distance mod 1 does not exceed $1 /(t+1)$. Putting $b=\left|r^{\prime}-r^{\prime \prime}\right|$ gives the required result.

University of CALIFornia 